\title{
About interactions between Banach-Lie groups and finite dimensional manifolds
}

\author{
By \\ Hideki OMORI and Pierre de la HaRPe \\ (Communicated by Professor Nagata, August 1971)
}

\section{Introduction}

Consider a smooth connected compact manifold without boundary, and let $\mathscr{D}$ be its group of diffeomorphisms. As shown in [14], $\mathscr{D}$ is a Frechet-Lie group. Furthermore, $\mathscr{D}$ is known to be a IHL-Lie group; this notion is a sort of generalization of Sobolev chains in function spaces. (Definition of IHL-Lie groups: see [6] or [16]; further properties in [17] and [18]; the present paper however uses neither the notion of a Frechet-Lie group, nor that of an IHL-Lie group). These two structures are rather difficult to study, because both the implicit function theorem and the Frobenius theorem are invalid for general Frechet or IHL manifolds.

For this reason, the following question arises naturally: "Does there exist a sufficienly large subgroup of $\mathscr{D}$ which carries a real Banach-Lie group structure?" The answer to this question is unfortunately negative, as this paper will show. Consequently, in order to study groups of diffeomorphisms, one must deal with Frechet-Lie groups or IHL-Lie groups, however difficult it might be.

Before we can state the theorem which answers the above question, we need a definition, to make precise the content of the words "sufficiently large". Let $G$ be a connected Banach-Lie group, with Lie algebra $\mathrm{g}$, which acts smoothly on a finite dimensional manifold $M$. 
Let $m_{0}$ be an arbitrary fixed point of $M$, and let $G_{0}$ be the isotropy subgroup of $G$ at $m_{0}$. The action of $G$ is said to be irreducible at $m_{0}$ if the linear representation $\rho$ of $G_{0}$ in $G L\left(T_{m_{0}} M\right)$ is irreducible, where $\rho(g)$ is the derivative of the map $m \mid \rightarrow g \cdot m$ at $m_{0}$ and where $T_{m_{0}} M$ is the tangent space to $M$ at $m_{0}$. Suppose now that $G_{0}$ is a sub Banach-Lie group and a submanifold of $G$, and let $g_{0}$ be its Lie algebra. (In fact $\mathfrak{g}_{0}$ will be defined page 7 without reference to $G_{0}$, so that no extra assumption on $G_{0}$ is needed.) The action is said to be primitive at $m_{0}$ if $g_{0}$ is maximal among the proper closed subalgebras of $\mathrm{g}$. If $G$ acts transitively on $M$, then the action is irreducible (respectively primitive) at any point of $M$ as soon as it is in some point. If so, we simply say that the action is irreducible (resp. primitive). Clearly, an irreducible action is always primitive, and many examples show that the converse does not hold. Finally, and in order to avoid restrictions implied by second countability arguments, we define a kind of "strong transitivity" as follows. Any vector in $\mathrm{g}$ defines a vector field on $M$, hence a tangent vector at the base point $m_{0}$ in $M$; the action is ample if it is transitive and if moreover this map from $\mathrm{g}$ to $T_{m_{0}} M$ is onto. Obviously, an irreducible action is ample as soon as the dimension of $M$ is 2 or more.

By a "sufficiently large" subgroup $G$ of $\mathscr{D}$, we mean a group $G$ which acts amply and primitively. However, the following result shows that $\mathscr{D}$ does not contain any such subgroup but finite dimensional ones.

Theorem I: Let $G$ be a connected Banach-Lie group acting smoothly, effectively and amply on a finite dimensional manifold without boundary. If the action is primitive, then $G$ must be a finite dimensional Lie group.

As a direct application of the method of proof, we have a second result.

Theorem II: Let $\mathrm{g}$ be the Lie algebra of an infinite dimen- 
sional Banach-Lie group G. Suppose g has no proper close finite codimensional ideal. Then $\mathrm{g}$ has no proper closed finite codimensional subalgebra. In particular any smooth action of $G$ on a finite dimensional manifold is trivial.

We introduce a convenient set up for these two theorems in section 2 , and show how they both follow from a property of primitiv graded Lie algebras, stated as theorem III (page 14). Theorem III itself is proved in section 3, by an elementary method resting on the classification of E. Cartan's real primitive infinite Lie algebras, that we know from the works of Morimoto and Tanaka [24]. (It has been pointed out to us that the classification is independently due to S. Schnider: J. of Diff. Geometry 4 (1970) 81-89.) The main idea of our proof appears in a simpler form in the two following examples.

Example. Let $\mathfrak{g}$ be the Lie algebra generated in the space of smooth functions from [01] to $\boldsymbol{R}$ by the elements

$$
\frac{\partial}{\partial x}, x \frac{\partial}{\partial x}, \cdots, x^{n} \frac{\partial}{\partial x}, \cdots
$$

$\mathrm{g}$ cannot be made a normed Lie algebra; indeed, the relation

$$
\left[x \frac{\partial}{\partial x}, x^{n} \frac{\partial}{\partial x}\right]=(n-1) x^{n} \frac{\partial}{\partial x}
$$

is not compatible with the existence of a bound $\kappa$ such that $\|[u, v]\|_{g}$ $\leqslant \kappa\|u\|_{\mathfrak{g}}\|v\|_{\mathfrak{g}}$ for all $u, v \in \mathrm{g}$. (Digression: Witt algebras give many examples of such infinite dimensional real or complex Lie algebras, where $\boldsymbol{R}$ and $\boldsymbol{C}$ are heuristically thought of as having characteristic infinity instead of zero).

Example. Let $\mathrm{g}$ be the Lie algebra generated in the space of smooth functions from [01] $\times$ [01] to $\boldsymbol{R}$ by the elements

$$
\frac{\partial}{\partial x}, \frac{\partial}{\partial y}, x \frac{\partial}{\partial y}, x^{2} \frac{\partial}{\partial y}, \cdots, x^{n} \frac{\partial}{\partial y}, \cdots
$$


Define a norm on $\mathfrak{g}$ by $\left\|a \frac{\partial}{\partial x}+\sum_{n \in \boldsymbol{N}} a_{n} x^{n} \frac{\partial}{\partial y}\right\|=|a|+\sum_{n \in \boldsymbol{N}} n !\left|a_{n}\right|$ where $a,\left(a_{n}\right)_{n \in \boldsymbol{N}}$ are real constants. Then one has

$$
\|[u, v]\|_{\mathfrak{g}} \leqslant 2\|u\|_{\mathfrak{g}}\|v\|_{\mathfrak{g}} \text {. }
$$

The crucial point is that $g$ does not contain any term of the form $x \frac{\partial}{\partial x}, y \frac{\partial}{\partial x}$ or $y \frac{\partial}{\partial y}$.

Indeed, we can develop the second example and show that the hypothesis of primitivity is essential in theorem I. This is the subject of section 4, where we prove that there exists a connected and infinite dimensional Banach-Lie group which acts smoothly, effectively and amply on the euclidian plane.

Theorem II applies in particular to the group $G L(H)$ of invertible bounded linear operators on a separable infinite dimensional Hilbert space, over the real or the complex field, and to various sub BanachLie groups of it. This is the object of the last section, which can be looked at as an infinite dimensional analogue of the following easy proposition: Let $\mathfrak{g}$ be a finite dimensional simple compact Lie algebra, of dimension $D$. Let $\mathfrak{b}$ be a non-trivial subalgebra of $\mathfrak{g}$, of codimension $d$. Then $d \geqslant \sqrt{2 D-\frac{1}{4}}+\frac{1}{2}$. Though we cannot state in this introduction a complete and precise version of the main result of section 5 , we give now a first approximation.

Theorem IV. Let $G(H)$ be a classical Banach-Lie group of bounded operators on a separable infinite dimensional Hilbert space $H$ over the reals or the complexes. Then the only possible smooth action of $G(H)$ on a finite dimensional manifold is the trivial action.

Theorem IV would naturally be trivial if $G(H)$ was replaced by a classical Banach-Lie group of compact operators in $H$, because its Lie algebra would then be simple (see Theorem II and end of section 5).

Finally, and though it is not directly relevant to the present 
paper, we recall a results of Eells, Burghelea and Kuiper which deals with a "dual" situation; namely, they have shown that "small" groups can always act "nicely" on infinite dimensional manifolds [2].

Result a. Let $G$ be a countably generated group and let $H$ be a separable infinite dimensional Hilbert space. Then there exists a free proparly discontinuous action of $G$ by smooth diffeomorphisms of $H$.

Result b. Let $G$ be a compact Lie group and let $H$ be as in Result a. Then there exists a free action with local smooth sections of $G$ by smooth diffeomorphisms of $H$.

The first author was supported by an SRC fellowship, and the "Fonds national suisse pour la Recherche scientifique". We thank them respectively, as well as I. Stewart for many conversations about the matter of section 5 , and $R$. Richardson, grace to whom it has been possible to correct a mistake in an earlier version of this work.

\section{Banach-Lie groups acting on finite dimensional manifolds}

\subsection{Primitive actions and maximal subalgebras}

We first "translate" some conditions given on an action in statements about subalgebras, and vice versa.

Let $G$ be conneted Banach-Lie group, with Lie algebra $g$, which acts smoothly, amply and primitively on a finite dimensional manifold $M$. Let $m_{0}$ be a fixed point in $M$ and let $G_{0}$ be the isotropy subgroup of $G$ at $m_{0}$. Let $f_{m_{0}}$ be the map from $G$ to $M$ which associates to an element $g$ in the group the transformed point $g \cdot m_{0}$ of the based point in the manifold. Let $d f_{m_{0}}$ be the derivative of this map at the identity of $G$, and put $\mathrm{g}_{0}=\operatorname{ker}\left(d f_{m_{0}}\right)$. By definition of amplicity, $d f_{m_{0}}(\mathrm{~g})=T_{m_{0}} M$. The implicit function theorem [4] implies that $G_{0}$ is a sub Banach-Lie group of $G$ with Lie algebra $g_{0}$, and a submanifold of $G$; and that the induced map $G / G_{0} \rightarrow M$ is a diffeomorphism. 
By definition of primitivity, $g_{0}$ is a maximal closed non-trivial subalgebra of g.

N. B.: Suppose that $G$ is second-countable and that the action is transitive. It is then a classical result that $G_{0}$ is a sub BanachLie group and a submanifold of $G$, and that $G / G_{0}$ is diffeomorphic to $M$; in particular, the action is ample. But we do not assume that $G$ is second-countable.

Suppose moreover that the action of $G$ is effective on $M$. It is then clear that $g_{0}$ cannot be an ideal in $g$, because if it was, the component of $G_{0}$ would be a non trivial normal subgroup of $G$; hence it would act trivially on $M \approx G / G_{0}$.

We sum up these considerations in the following proposition.

Proposition 2.1: Suppose the action of $G$ satisfies the conditions of Theorem $I$. Then $G_{0}$ is a submanifold and a sub BanachLie group of $G$, whose Lie algebra $\mathrm{g}_{0}$ is a maximal closed nontrivial subalgebra of $\mathrm{g}$; moreover, $\mathrm{g}_{0}$ is not an ideal in $\mathrm{g}$.

Let now $g$ be the Lie algebra of some connected Banach-Lie groups $G$, and let $g_{0}$ be a maximal closed proper subalgebra of finite codimension in $\mathrm{g}$, which is not an ideal in $\mathrm{g}$. Let $\mathrm{m}$ be a complementary subspace of $g_{0}$ in $g$.

Lemma 2. 2: There exists a neighbourhood $V(r e s p . W)$ of the origin in $\mathrm{g}_{0}$ (resp. $\mathrm{m}$ ) and a smooth diffeomorphism $\Phi$ of $V \oplus W$ onto a neighbourhood $U$ of the identity $e$ in $G$ such that

i) The derivative $d \Phi_{0}$ of $\Phi$ at the origin is the identity

ii) For each $w \in W, \emptyset(V \oplus\{w\})$ is an integral submanifold of $\ddot{\mathrm{g}}_{0}$, where $\tilde{\mathrm{g}}_{0}$ is the left-invariant distribution defined by $\mathrm{g}_{0}$ on $G$.

Proof: We recall that $g_{0}$ is the collection of the images of $\mathrm{g}_{0}$ by all the $d L_{\mathrm{g}}$ 's, where $d L_{\mathrm{g}}$ is the derivative at the identity of $G$ of the left multiplication by $g$ in $G$, for all $g \in G$. Lemma 2.2 is a direct consequence of Frobenius theorem [4]. 
Let now $G_{0}$ be the maximal integral submanifold of $\tilde{\mathrm{g}}_{0}$ through the identity of $G$. $G_{0}$ is a connected sub Banach-Lie group of $G$. Because of the maximality of $g_{0}$, one has:

Lemma 2. 3: (Notation as in 2.2) The neighbourhood $W$ can be chosen such that $\Phi(\{0\} \oplus W)$ and $G_{0}$ intersect only at the identity of $G$; therefore, $G_{0}$ is a closed subgroup of $G$.

Proof: (ab absurdo) Suppose there exists a sequence $\left(x_{n}\right)_{n \in N}$ im $\mathrm{m}$ of non-zero vectors such that $\emptyset\left(x_{n}\right) \in G_{0}$ for all $n \in N$. Taking a subsequence if necessary, we may assume that the sequence $\left(\frac{x_{n}}{\left\|x_{n}\right\|}\right)_{n \in N}$ converges to some element $X$ in $\mathfrak{m}$. By a simple and careful argument we can choose a $\mathcal{C}^{1}$-curve $t \mid \rightarrow c(t)$ in $m$ such that the image of the curve contains infinitely many points of $\left(x_{n}\right)_{n \in N}$. Taking again a subsequence, we may assume that for each $n \in N$, there is a value $t_{n}$ of the parameter with $c\left(t_{n}\right)=x_{n}$. Obviously, $\lim _{n \rightarrow \infty} t_{n}=0$. Now $\emptyset\left(x_{n}\right) \in G_{0}$ implies $A d\left(\Phi\left(c\left(t_{n}\right)\right)\right) g_{0} \subset \mathfrak{g}_{0}$ for all $n \in \boldsymbol{N}$; so that $\left.\frac{d}{d t}\right|_{t=0} \operatorname{Ad}(\Phi(c(\cdot))) \mathfrak{g}_{0} \subset \mathrm{g}_{0}$. On the other hand, $\left.\frac{d}{d t}\right|_{t=0}$ $\operatorname{Ad}(\Phi(c(\cdot))) \mathrm{g}_{0}=a d\left(d \Phi_{0}(X)\right) \mathrm{g}_{0}=\left[X, \mathrm{~g}_{0}\right]$. Hence $\boldsymbol{R} X \oplus \mathrm{g}_{0}$ is a subalgebra of $\mathrm{g}$; by maximality, $\boldsymbol{R} X \oplus \mathrm{g}_{0}=\mathrm{g}$ and $\mathrm{g}_{0}$ is an ideal in $\mathrm{g}$, which contradicts the hypothesis.

Defining $M$ to be the homogeneous space $G / G_{0}$, we obtain the following statement.

Proposition 2.4: Let $\mathrm{g}$ be the Lie algebra of some connected Banach-Lie group $G$. Let $\mathrm{g}_{0}$ be a maximal closed proper subalgebra of finite codimension in $\mathrm{g}$, which is not an ideal in $\mathrm{g}$. Then $\mathrm{g}_{0}$ is the Lie algebra of a closed sub Banach-Lie group and submanifold $G_{0}$ of $G$; the natural action of $G$ on $G / G_{0}$ is smooth, ample and primitive.

\section{2. Filtered and graded Lie algebras associated to an action}

Let $\mathscr{F}$ be the space of formal power series of local vector fields 
at the origi of $\boldsymbol{R}^{n}$, i.e. of expressions of the form $\sum_{i=1}^{n} \sum_{\alpha \in N^{n}} A_{\alpha}^{i} x^{\alpha} \frac{\partial}{\partial x^{i}}$ where the $A_{\alpha}^{i}$ 's are real constants (we use the classical notation for multi-indices; see e.g. [4]). $\Psi_{4}$ is clearly an infinite dimensional real vector space, and a Lie algebra with the evident Lie bracket. Define

$$
J_{k}=\left\{P \in \mathscr{F} \mid P=\sum_{i=1}^{n} \sum_{\substack{\alpha \in N^{n} \\|\alpha|=k+1}} A_{\alpha}^{i} x^{\alpha} \frac{\partial}{\partial x_{i}}\right\}
$$

and

$$
F_{k}=\left\{P \in \mathscr{I} \mid P=\sum_{i=1}^{n} \sum_{\substack{\alpha \in N^{n} \\|\alpha|=k+1}} A_{\alpha}^{i} x^{\alpha} \frac{\partial}{\partial x_{i}}\right\}
$$

for each $k=-1,0,1, \cdots$. It is straightforward to check that $\left\{J_{k}\right\}_{k=-1,0,1, \ldots}$ makes $\mathscr{I}$ a filtered Lie algebra and that the subspace $F=\bigoplus_{k=-1}^{\infty} F_{k}$ is the associated graded Lie algebra. (For all notions concerning filtered and graded Lie algebras, the reader is referred to the usual literature: [23] or $[11,12]$ and references there. Note that $\mathscr{F}=\prod_{k=-1}^{\infty} F_{k}$, where $\amalg$ denotes the direct product, or "complete direct sum"; see [12] p. 164). If $M$ is a $n$-dimensional manifold, if $m_{0}$ is a fixed point in $M$, and if $\left(x_{1}, \cdots, x_{n}\right)$ is a system of coordinates centered at $m_{0}$, F can be considered as the Lie algebra of formal power series of local vector fields at $m_{0}$ to $M$.

Let now $G$ be a connected Banach-Lie group, with Lie algebra $g$, which acts smoothly on $M$, and suppose the action is ample and primitive at $m_{0}$. Any $X \in \mathrm{g}$ defines a smooth vector field on $M$ which takes at $m \in M$ the value $\left.\frac{d}{d t}\right|_{t=0}(\exp t X)(m)$. Let $\widehat{X}$ be the Taylor series of this vector field at $m_{0} ; \widehat{X} \in \mathcal{F}$. This construction provides a map $\Psi: g \rightarrow \mathscr{F}$ which is clearly a Lie algebra homomorphism such that $g_{0}=\Psi^{-1}\left(J_{0}\right)$. Define $g_{k}=\Psi^{-1}\left(J_{k}\right)$ for all $k=-1,0,1$, ... Then $\mathfrak{h}=\bigcap_{k=-1}^{\infty} \mathrm{g}_{k}$ is a closed ideal in $\mathrm{g}$. Define $\mathcal{E}=\mathrm{g} / \mathrm{h}$ and $I_{k}=\mathrm{g}_{k} / \mathfrak{h}$ for $k=-1,0,1, \cdots$. The family $\left\{I_{k}\right\}_{k=-1,0,1, \ldots}$ makes $\mathcal{E}$ a filtered Lie algebra and the map $\Psi$ induces an injective morphism $\chi: \mathcal{E} \rightarrow \mathscr{F}$. 
Lemma 2. 5: $\mathcal{E}$ is a primitive Banach filtered Lie algebra and there exists an inclusion $\chi$ of $\mathcal{E}$ in $\mathcal{F}$.

Proof: As the action of $G$ on $M$ is ample at $m_{0}$, we have $\operatorname{dim} \mathrm{g} / \mathrm{g}_{0}=\operatorname{dim} \mathcal{E} / I_{0}=\operatorname{dim} M$, and $\mathrm{g}_{0}$ is maximal in $\mathrm{g}$ by primitivity. As $g$ is a Banach-Lie algebra, so is $\mathcal{E}$ for the norm defined by $\|\bar{u}\|=\inf _{u \in \bar{u}}\|u\|_{\mathfrak{g}}$ for all $\bar{u} \in \mathcal{E}$. To say that $\chi$ is an inclusion is to say that $\chi$ is an injective morphism in the category of filtered Lie algebras over $\boldsymbol{R}$ which preserves the degree.

Define $E_{k}=I_{k} / I_{k+1}$ for $k=-1,0,1, \cdots$ and let $E=\bigoplus_{k=-1}^{\infty} E_{k}$ be the graded Lie algebra associated to the filtered Lie algebra $\mathcal{E}$. $\chi$ induces an inclusion $E \rightarrow F$ which we will abusively denote by the same letter.

Proposition 2. 6: $E$ is a normed graded Lie algebra and there exists an inclusion $\chi$ of $E$ in $F$; moreover, $\operatorname{dim} E_{-1}=\operatorname{dim} F_{-1}$. ( $E$ may or may not be irreducible.)

Proof: If each $E_{k}$ is given the quotient norm from $I_{k} / I_{k+1}$ and if one defines $\|v\|=\sum_{k=-1}^{\infty}\left\|v_{k}\right\|$ for each $v=\sum v_{k} \in E$, it is easy to check that $\left\|\left[v, v^{\prime}\right]\right\| \leqslant \operatorname{cste}\|v\|\left\|v^{\prime}\right\|$ for all $v, v^{\prime} \in E$; so that $E$ is normed. Finally one has $\operatorname{dim} E_{-1}=\operatorname{dim} I_{-1} I_{0}=\operatorname{dimg} / \mathfrak{g}_{0}=\operatorname{dim} M=\operatorname{dim} F_{-1}$.

In the case $\mathcal{E}$ is irreducible, $E$ will be the convenient object to consider in order to prove the result we need (Theorem III below). The other case however requires one more construction that we are going to sketch now.

Suppose $\mathcal{E}$, or more precisely the couple $\left(\mathcal{E}, I_{0}\right)$, is not irreducible. According to [24], it is possible to define a new filtration called the irreducible filtration of $\left(\mathcal{E}, I_{0}\right)$ as follows. Let $H_{-1}$ be a minimal subspace of $\mathcal{E}$ such that $H_{-1} \supsetneq I_{0}$ and such that $\left[I_{0}, H_{-1}\right] \subset H_{-1}$. For any $k \in Z, k \neq-1$, define now $H_{k}$ by:

$$
H_{0}=I_{0}
$$




$$
\begin{array}{lll}
H_{k}=\left[H_{-1}, H_{k+1}\right]+H_{k+1} & \text { if } & k<-1 \\
H_{k}=\left\{X \in H_{k-1} \mid\left[X, H_{-1}\right] \subset H_{k-1}\right\} & \text { if } & k>0 .
\end{array}
$$

Then on has (Lemma 1.1 in [24]):

$$
\begin{aligned}
& \text { i) }\left[H_{p}, H_{q}\right] \subset H_{p+q} \quad \text { for all } p, q \in \boldsymbol{Z} \\
& \text { ii) } \mathcal{E}=H_{-\mu} \quad \text { for some } \mu>0 \\
& \text { iii) } H_{p} \supset I_{p} \supset H_{\mu p} \quad \text { for all } p \in \boldsymbol{N} \\
& \text { iv) } \bigcap_{p=-\mu}^{+\infty} H_{p}=\{0\} \text {. }
\end{aligned}
$$

Define now $C_{k}=H_{k} / H_{k+1}$ for all $k \in Z, k \geqslant-\mu$ and let $C=\bigoplus_{k=-\mu}^{+\infty} C_{k}$ be the graded Lie algebra associated to the irreducible filtration of $\mathcal{E}$. The same procedure as above endowes $C$ with a norm, so that we have:

Proposition 2. 7: $\quad C$ is a normed irreducible graded Lie algebra.

Note first that if $\left(\mathcal{E}, I_{0}\right)$ had been irreducible to start with, then the filterations given by the $H$ 's and by the $I$ 's would coincide, and $C$ would be identical to $E$. Secondly, if $\left(\mathcal{E}, I_{0}\right)$ is not irreducible, we do not need to precise a map which would correspond to the map $\chi$ of Proposition 2.6. Finally, let us remark that to mimick [24] precisely, we should have taken the completion of $\mathcal{E}$ with respect to the linear uniform structure defined by the filtration $\left(H_{k}\right)_{k \in \boldsymbol{Z}}$. However, the graded Lie algebra defined by this completion of $\mathcal{E}$ is identical with $H$; it follows that, for our purpose, the technical step of the completion can be omitted.

\section{3. Proof of Theorems I and II, modulo Theorem III}

Theorem III: Let $\mathcal{E}$ be a primitive filtered normed Lie algebra. Suppose that there exists an inclusion of $\mathcal{E}$ into $\mathscr{I}$ and that $\operatorname{dim} E_{-1}=\operatorname{dim} F_{-1} . \quad$ Then $\operatorname{dim} \mathcal{E}<\infty$.

The proof of this theorem is the object of the following section. 
Theorem III implies Theorem I. Suppose $G$ is acting on $M$ according to the hypotheses of Theorem I. Let $g, G_{0}$ and $g_{0}$ be as in Proposition 2.1. Define $\mathcal{E}$ as before Lemma 2.5. Assuming Theorem III is true, $\operatorname{dim} \mathcal{E}<\infty$ and $\mathfrak{h}$ is a finite codimensional ideal in g. By Frobenius theorem [4], there is a normal subgroup $N$ in $G$ with Lie algebra $\mathfrak{h}$, and $N$ is evidently contained in $G_{0}$. Since $G$ acts effectively on $M$, one must have $N=\{e\}$, i.e. $\mathfrak{h}=\{0\}$. Consequently $\operatorname{dim} g<\infty$.

Theorem III implies Theorem II. Let $\mathfrak{g}$ be a Banach-Lie algebra which satisfies the hypothesis of Theorem II. Suppose $\mathfrak{g}$ has proper closed subalgebras of finite codimension; choose a maximal one, say $g_{0}$. Remembering Proposition 2.4, we can apply the same argument as above to show that $\mathrm{g}$ has proper closed ideal of finite codimension. This contradicts the hypothesis and ends the proof.

\section{Primitive filtered normed Lie algebras}

This section is the proof of Theorem III. Keeping in mind how A. Borel read G. B. Show, we use E. Cartan's classification of the primitive filtered Lie algebras of infinite type; see [12], mainly their Theorem II, and [23], Theorem 5.1, for the irreducible case; see [24] for the general case.

Let us first assume that $\mathcal{E}$ is an irreducible filtered normed Lie algebra. Then $E$ is by definition an irreducible normed graded Lie algebra over $R$, contained in the algebra $F$ defined in 2.2, and such that $E_{-1}=F_{-1}$. By definition:

$$
F_{-1}=\left\{P \in \mathscr{F} \mid P=\sum_{i=1}^{n} a_{i} \frac{\partial}{\partial x_{i}}\right\} \text { and } F_{0}=\left\{P \in \mathscr{F} \mid P=\sum_{i, j=1}^{n} a_{i j} x_{i} \frac{\partial}{\partial x_{j}}\right\} .
$$

Thus $F_{-1}$ can be naturally identified with $\boldsymbol{R}^{n}$ and $F_{0}$ with $\mathfrak{g l}\left(\boldsymbol{R}^{n}\right)$. Therefore, $E_{0}$ is a sub-Lie algebra of $\operatorname{gl}\left(\boldsymbol{R}^{n}\right)$ whose natural representation in $\boldsymbol{R}^{n}$ is irreducible.

Let $E^{c}$ be the complexification of $E$, and $\mathscr{F}^{c}$ that of $\mathscr{F} \cdot E^{c}$ $=\bigoplus_{k=-1}^{\infty} E_{k}^{C}$ is a normed graded Lie algebra over $C$ contained in $\mathscr{F}^{C}$. 
Clearly $F_{0}^{C}=\mathfrak{g l}\left(C^{n}\right)$, but the representation of $E_{0}^{C}$ in $\boldsymbol{C}^{n}$ may or may not be irreducible. We treat the two cases successively.

\section{1. The case when $E^{C}$ is irreducible}

Assume that $E^{C}$ is of in, infinite type. As a result of the classification referred to above, the sub Lie algebra $E_{0}^{C}$ of $F_{0}^{C}=\mathfrak{g l}\left(C^{n}\right)$ can only be one of the four following ones (after a change of coordinates if necessary):

(1) $E_{0}^{C}=\mathfrak{g l}\left(\boldsymbol{C}^{n}\right)$

(2) $E_{0}^{C}=\mathfrak{g l}\left(C^{n}\right)=\left\{A \in \mathfrak{g l}\left(C^{n}\right) \mid \operatorname{trace}(A)=0\right\}$

(3) $E_{0}^{C}=\mathfrak{g p}(n, C)=\left\{A \in \mathfrak{g l}\left(C^{n}\right) \mid A=\left(\begin{array}{cc}Z_{1}, & Z_{2} \\ Z_{3}-{ }^{t} Z_{1}\end{array}\right)\right.$ with the $Z_{i}$ complex $m \times m$ matrices $(i=1,2,3)$ and $Z_{2}, Z_{3}$ symmetric\} where $n=2 m$.

(4) $E_{0}^{G}=\mathfrak{g p}(n, \boldsymbol{C}) \oplus \boldsymbol{C} i d$, where $i d$ is the $n \times n$ unit matrix.

Moreover, $E^{c}$ is normed; let $\kappa$ be a constant such that $\|[u, v]\|$ $\leqslant \kappa\|u\|\|v\|$ for all $u, v \in E^{c}$. Using this hypothesis, we are going to exclude one after the other the four possibilities listed above.

Lemma 3. 1: $E_{0}^{c}$ cannot have a non-trivial center. So that the cases (1) and (4) of the list are to be excluded.

Proof: Suppose $E_{0}^{C}$ has a non-trivial centre. Then $E_{0}^{C}$ contains the element $\sum_{i=1}^{n} x_{i} \frac{\partial}{\partial x_{i}}$. Let $k \in N$ and let $\sum_{i=1}^{n} \sum_{\substack{\alpha \in N^{n} \\|\alpha|=k+1}} A_{\alpha}^{i} x^{\alpha} \frac{\partial}{\partial x_{i}}$ be a nonzero element in $E_{k}^{C}$. By direct computation:

$$
\left[\sum x_{i} \frac{\partial}{\partial x_{i}}, \sum A_{\alpha}^{i} x^{\alpha} \frac{\partial}{\partial x_{i}}\right]=k \sum A_{\alpha}^{i} x^{\alpha} \frac{\partial}{\partial x_{i}} .
$$

This being true for arbitrary large $k$ 's, it makes impossible an identity of the form $\|[u, v]\| \leqslant \kappa\|u\|\|v\|$ for all $u, v \in E^{c}$.

Lemma 3. 2: $E_{0}^{C}$ cannot be $\mathfrak{g l}\left(\boldsymbol{C}^{n}\right)$.

Proof: Suppose $E_{0}^{\boldsymbol{C}}=\mathfrak{g l}\left(\boldsymbol{C}^{n}\right)$; let $k$ be an arbitrary integer.

Step one: $E_{k}^{C}$ contairs a non-zero element of the form 
$p=\sum_{i=2}^{n} \sum_{\substack{|\beta|=k+1 \\ \beta \in N^{n}}} a_{\beta}^{i} x^{\beta} \frac{\partial}{\partial x_{i}}$.

Choose a non-zero element $\sum_{i=1}^{n} \sum_{|\alpha|=k+1} A_{\alpha}^{i} x^{\alpha} \frac{\partial}{\partial x_{i}} \in E_{k}^{C}$. Assume that $A_{\alpha}^{1} \neq 0$ for some $\alpha \in N^{n},|\alpha|=k+1$. Let $j \in\left\{2,3, \cdots, n_{5} ; x_{1} \frac{\partial}{\partial x_{j}} \in E_{0}^{c}\right.$ $=\mathfrak{g l}\left(\boldsymbol{C}^{n}\right)$. By direct computation: $\left[x_{1} \frac{\partial}{\partial x_{j}}, \sum_{i, \alpha} A_{\alpha}^{i} x^{\alpha} \frac{\partial}{\partial x_{i}}\right]=\sum_{\substack{|\alpha|=k+1 \\ \alpha_{j} \neq 0}} \alpha_{j} A_{\alpha}^{1}$

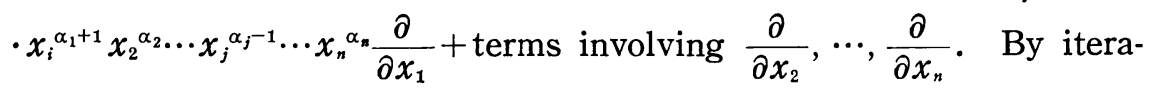
tion, one obtains an element of the form

$$
P^{\prime}=a x_{1}{ }^{k+1} \frac{\partial}{\partial x_{1}}+\sum_{i=2}^{n} \sum_{\substack{\alpha \in N^{n} \\|\alpha|=k+1}} B_{\alpha}^{i} x^{\alpha} \frac{\partial}{\partial x_{i}},
$$

where $a$ is a non-zero complex constant.

$$
\left[x_{1} \frac{\partial}{\partial x_{2}}, P^{\prime}\right]=-a x_{1}^{k+1} \frac{\partial}{\partial x_{2}}+\sum_{i, \alpha} * \frac{\partial}{\partial x_{i}} .
$$

If this last expression is not zero, we have established step one. If it is zero then

$$
P^{\prime}=a x_{1}{ }^{k+1} \frac{\partial}{\partial x_{1}}+a x_{1}^{k} x_{2} \frac{\partial}{\partial x_{2}}+\sum_{i=3}^{n} \sum_{\substack{|\alpha|=k+1 \\ \alpha 2=0}} C_{\alpha}^{i} x^{\alpha} \frac{\partial}{\partial x_{i}}
$$

and the third term is not zero; indeed, if it was it would imply

$$
\left(a d \frac{\partial}{\partial x_{1}}\right)^{k}\left(P^{\prime}\right)=a(k+1) ! x_{1} \frac{\partial}{\partial x_{1}}+a k ! x_{2} \frac{\partial}{\partial x_{2}} \in E_{0}^{C}=\mathfrak{H l}\left(\boldsymbol{C}^{n}\right),
$$

i.e. $a=0$, which is impossible. Write

$$
P^{\prime}=a x_{1}^{k+1} \frac{\partial}{\partial x_{1}}+a x_{1}^{k} x_{2} \frac{\partial}{\partial x_{2}}+P^{\prime \prime} .
$$

Then

$$
\left[x_{1} \frac{\partial}{\partial x_{3}}, P^{\prime}\right]=-a x_{1}{ }^{k+1} \frac{\partial}{\partial x_{3}}+\left[x_{1} \frac{\partial}{\partial x_{3}}, P^{\prime \prime}\right] .
$$

If this last expression not zero, we have established step one. If it is zero then

$$
P^{\prime}=a x_{1}{ }^{k+1} \frac{\partial}{\partial x_{1}}+a x_{1}^{k} x_{2} \frac{\partial}{\partial x_{2}}+a x_{1}^{k} x_{3} \frac{\partial}{\partial x_{3}}+\sum_{\substack{i=4 \\ \text { a }}}^{n} \sum_{\substack{\alpha=k+1 \\ \alpha_{2}=\alpha_{3}=0}} D_{\alpha}^{i} x^{\alpha} \frac{\partial}{\partial x_{i}} .
$$

By iteration of the procedure, one finds either an element of the 
desired form, or that

$$
P^{\prime}=a x_{1}^{k+1} \frac{\partial}{\partial x_{1}}+a x_{1}^{k} x_{2} \frac{\partial}{\partial x_{2}}+\cdots+a x_{1}^{k} x_{n} \frac{\partial}{\partial x_{n}} .
$$

The second possibility cannot happen, because if it $\operatorname{did}:\left(\operatorname{ad} \frac{\partial}{\partial x_{1}}\right)^{k}\left(P^{\prime}\right)$ $\in \mathfrak{E l}\left(\boldsymbol{C}^{n}\right)$ would imply $a=0$, which is impossible.

Step two: $E_{k}^{C}$ contains a non-zero element of the form $\sum_{i=2}^{n} a_{i} x_{1}{ }^{k+1} \frac{\partial}{\partial x_{i}}$. Indeed: start with an element $P=\sum_{i=2}^{n} \sum_{\beta} a_{\beta}^{i} x^{\beta} \frac{\partial}{\partial x_{i}}$ and apply $a d\left(x_{1} \frac{\partial}{\partial x_{j}}\right)$ to $P$ conveniently many times, for $j=2,3 \cdots, n$; the result is an element of the desired form.

End of proof: Let $P=\sum_{i=2}^{n} a_{i} x_{1}{ }^{k+1} \frac{\partial}{\partial x_{i}} \in E_{k}^{c}, P \neq 0$

$$
\left[x_{1} \frac{\partial}{\partial x_{1}}-x_{2} \frac{\partial}{\partial x_{2}}, P\right]=(k+2) a_{2} x_{1}{ }^{k+1} \frac{\partial}{\partial x_{2}}+(k+1) \sum_{i=3}^{n} a_{i} x_{1}{ }^{k+1} \frac{\partial}{\partial x_{i}} .
$$

If $a_{2}=0$ then

$$
\left[x_{1} \frac{\partial}{\partial x_{1}}-x_{2} \frac{\partial}{\partial x_{2}}, P\right]=(k+1) P
$$

If $a_{2} \neq 0$ then

$$
\left[x_{1} \frac{\partial}{\partial x_{1}}-x_{2} \frac{\partial}{\partial x_{2}}, P\right]=a_{2} x_{1}^{k+1} \frac{\partial}{\partial x_{2}}+(k+1) P
$$

hence $x_{1}{ }^{k+1} \frac{\partial}{\partial x_{2}} \in E_{k}^{C}$ and

$$
\left[x_{1} \frac{\partial}{\partial x_{1}}-x_{2} \frac{\partial}{\partial x_{2}}, x_{1}{ }^{k+1} \frac{\partial}{\partial x_{2}}\right]=(k+2) x_{1}{ }^{k+1} \frac{\partial}{\partial x_{2}} .
$$

Now we make use of the hypothesis that $E^{c}$ is normed; both alternatives can be excluded by the same argument as that used for proving Lemma 3. 1 .

Lemma 3. 3: $E_{0}^{C}$ cannot be $\mathfrak{s p}(n, C)$.

Proof: Suppose $E_{0}^{C}=s p(n, C) n=2 m$. Put $y_{i}=x_{m+i}$ for $i=1$, $2, \cdots, m$. Note that $E_{0}^{C}$ contains $\sum_{i=1}^{m} x_{i} \frac{\partial}{\partial x_{i}}-\sum_{j=1}^{m} y_{j} \frac{\partial}{\partial y_{j}}$. Let $k$ be an arbitrary integer. 
Step one: $E_{k}^{C}$ contains a non-zero element of the form

$$
P=\sum_{j=1}^{m} \sum_{\substack{\alpha, \beta \in N_{N}^{m} \\|\alpha|+|\beta|=k+1}} a_{\alpha \beta}^{j} x^{\alpha} y^{\beta} \frac{\partial}{\partial y_{j}} .
$$

Choose a non-zero element

$$
Q=\sum_{i=1}^{m} \sum_{\alpha, \beta} A_{\alpha, \beta}^{i} x^{\alpha} y^{\beta} \frac{\partial}{\partial x_{i}}+\sum_{j=1}^{m} \sum_{\gamma, \delta} B_{\gamma \delta}^{j} x^{\gamma} y^{\delta} \frac{\partial}{\partial y_{j}}
$$

in $E_{k}^{c}$. Assume $A_{\alpha \beta}^{i} \neq 0$ for some $\alpha, \beta \in N^{m}, i \in\{1, \cdots, m\}$. Apply $a d\left(x_{l} \frac{\partial}{\partial y_{l}}\right)$ to $Q$ conveniently many times $(l=1, \cdots, m)$ to get an element

$$
Q^{\prime}=\sum_{i=1}^{m} \sum_{\alpha} a_{\alpha}^{i} x^{\alpha} \frac{\partial}{\partial x_{i}}+\sum_{j=1}^{m} \sum_{\gamma \delta} C_{\gamma \delta}^{j} x^{\gamma} y^{\delta} \frac{\partial}{\partial y_{j}}
$$

in $E_{k}^{C}$ and not zero. Now

$$
\left[x_{l} \frac{\partial}{\partial y_{l}}, Q^{\prime}\right]=\left(-\sum_{\alpha} a_{\alpha}^{l} x^{\alpha}\right) \frac{\partial}{\partial y_{l}}+\sum_{j=1}^{n} * \frac{\partial}{\partial y_{j}} .
$$

If this last expression is not zero for some $l \in\{1, \cdots, m\}$, we have established step one. If it is zero for any $l \in\{1, \cdots, m\}$ then

$$
Q^{\prime}=\sum_{i=1}^{n} \sum_{\alpha} a_{\alpha}^{i} x^{\alpha} \frac{\partial}{\partial x_{i}}+\sum_{j=1}^{n} \sum_{\substack{\gamma \mid \gamma=k+1 \\ \gamma \neq \neq 0}} a_{\gamma}^{j} \frac{x^{\gamma}}{x_{j}} y_{j} \frac{\partial}{\partial y_{j}} .
$$

Apply $k$ times $a d\left(\frac{\partial}{\partial x_{l}}\right)$ for $l$ judicously chosen in $\{1, \cdots, m\}$ and obtain

$$
Q^{\prime \prime}=\sum_{i=1}^{n} a^{i} x_{i} \frac{\partial}{\partial x_{i}}+\sum_{j=1}^{n} a^{j} y_{j} \frac{\partial}{\partial y_{j}} \in \mathfrak{S p}(n, C),
$$

which is impossible.

Step two: $E_{k}^{C}$ contains a non-zero element of the form

$$
P^{\prime}=\sum_{j=1}^{n} \sum_{\alpha \in N^{n}} A_{\alpha}^{j} x^{\alpha} \frac{\partial}{\partial y_{j}} .
$$

This is easily seen by applying $a d\left(x_{t} \frac{\partial}{\partial y_{l}}\right)$ enough times to the element $P$ constructed in step one.

End of proof: Let $P_{0}=\sum_{i=1}^{m} x_{i} \frac{\partial}{\partial x_{i}}-\sum_{j=1}^{m} y_{j} \frac{\partial}{\partial y_{j}} \in E_{0}^{C}$ 
and let $P^{\prime}$ be the element constructed in step two. Then $\left[P_{0}, P^{\prime}\right]$ $=(k+1) P^{\prime}$. The end of proof is as in the previous lemma.

These three lemma exclude the irreducible case.

\section{2. The case when $E^{C}$ is reducible ( $E$ irreducible)}

If $E^{C}$ is reducible, the dimension of $E_{-1}$ is even, say equal to $2 m ; E_{-1}^{C}$ splits into two $m$ dimensional spaces $V$ and $\bar{V}$, and $E_{0}^{C}$ acts irreducibly on each of them. By a suitable linear change of coordinates one can arrive at the following situation: $E_{-1}^{C_{1}}=F_{-1}^{C}$ is described by coordinates $\left(x_{1}, \cdots, x_{m}, y_{1}, \cdots, y_{m}\right)$; put $z_{i}=x_{i}+\sqrt{-1} y_{i}$ and $\bar{z}_{i}=x_{i}-\sqrt{-1} y_{i}$ for $1 \leqslant i \leqslant m$, so that

$$
\frac{\partial}{\partial z_{i}}=\frac{1}{2}\left(\frac{\partial}{\partial x_{i}}-\sqrt{-1} \frac{\delta}{\delta y_{i}}\right) \text { and } \frac{\partial}{\partial \bar{z}_{i}}=\frac{1}{2}\left(\frac{\partial}{\partial x_{i}}+\sqrt{-1} \frac{\partial}{\partial y_{i}}\right)
$$

then $V$ is spanned by the $\frac{\partial}{\partial z_{i}}$ 's and $\bar{V}$ by the $\frac{\partial}{\partial \bar{z}_{i}}$ 's. $E_{0}^{C}$ splits into the direct sum of two sub Lie algebras of $F_{0}^{C}=\mathfrak{g l}\left(\boldsymbol{C}^{n}\right): E_{0}^{\boldsymbol{C}}$ $=V E_{0} \oplus \overline{V E}_{0}$; any element in $V E_{0}$ (resp. in $\overline{V E}_{0}$ ) can be written under the form $\sum_{i, j=1}^{m} a_{i j} z_{i} \frac{\partial}{\partial z_{j}}$ (resp. $\sum_{i, j=1}^{m} b_{i j} \bar{z}_{i} \frac{\partial}{\partial \bar{z}_{j}}$ ) where the $a_{i j}$ 's (resp. $b_{i j}$ 's) are some complex constants. These facts can be checked either using a general theory of complexification, or case by case (six of them) using the classification of irreducible real graded Lie algebras ([23] Th. 5.2 or [12] section 10).

Let $P$ be an arbitrary element in $E_{k}^{C} ; P$ is of the form

$$
\sum_{i=1}^{m} \sum_{|\alpha|=k+1} A_{\alpha}^{i} z^{\alpha} \frac{\partial}{\partial z_{i}}+\sum_{j=1}^{m} \sum_{|\beta|=k+1} B_{\beta}^{j} \bar{z}^{\beta} \frac{\partial}{\partial \bar{z}_{j}} .
$$

Indeed, it is true for $k=0$; an induction argument consisting of looking at $\left[\frac{\partial}{\partial z_{i}}, P\right]$ and $\left[\frac{\partial}{\partial \bar{z}_{j}}, P\right]$ shows that it is true for any $k$. Define $\overline{V E}_{k}=\left\{P \in F_{k}^{C} \mid[V, P]=0\right\}$ for all $k \in N$, and $\overline{V E}=\bigoplus_{k=-1}^{\infty} \overline{V E}_{k}$. $\overline{V E}$ is an irreducible complex graded Lie algebra, which is clearly normed and included in a graded Lie algebra of formal polynomials of local vector fields. By 3.1, one has $\operatorname{dim} \overline{V E}<\infty$. Define now 
$V E_{k}=E_{k}^{C} / \overline{V E_{k}} ; \quad V E_{k}$ is normed for the quotient norm, and $V E$ $=\bigoplus_{k=1-1}^{\infty} V E_{k}$ is an irreducible complex graded Lie algebra. Again, there is clearly an inclusion map from $V E$ into an algebra of formal polynomials of vector fields. Hence $\operatorname{dim} V E<\infty$, and $\operatorname{dim} E^{c}<\infty$.

This completes the proof of Theorem III in the special case where the graded algebra $E$ is irreducible.

\section{3. The contact case}

Finally, we have to consider the case where $\left(\mathcal{E}, I_{0}\right)$ is a primitive filtered Lie algebra over $R$, not irreducible and of infinite type. According to the classification of Morimoto and Tanaka [24], the associated graded Lie algebra $H$ (as in Proposition 2.7) can only be one of the two following:

i) the real contact Lie algebra

ii) the complex contact Lie algebra regarded as a real algebra. Both cases can be defined as the prolongation (in the sense of [24]) of a Lie algebra $c_{-2} \oplus c_{-1}$, where $c_{-2}$ is of dimension 1 and $c_{-1}$ is of finite even dimension (over the appropriate field). As $\left[c_{-1}, c_{-1}\right] \subset c_{-2}$, the bracket defines a bilinear form on $c_{-1}$ which is non-degenerate.

$c=\bigoplus_{k=-2}^{+\infty} c_{k}$ be the prolongation of $c_{-2} \oplus c_{-1}$. There is a non-zero element $A$ in the center of $c_{0}$, uniquely determined, such that $[A, X]$ $=k X$ for all $k \in Z$, for all $X \in c_{k}$ (see [24], section 3.2). As before (see for example our Lemma 2.1), this is incompatible with the existence of a norm on the graded algebra $c$.

The proof of Theorem III is now completed.

\section{An infinite dimensional Banach-Lie group which acts smooth- $l y$, effectively and transitively on $R^{2}$}

Let $P$ be the space of analytic real functions of a real variable whose Taylor series at the origin is of the form $f(x)=\sum_{n=0}^{\infty} a_{n} x^{n}$ with $\sup _{n \in N}\left|a_{n} n !\right|<\infty$. (In particular, the radius of convergence of this series is infinite). $P$ is a real Banach space for the norm defined by 
$\|f\|=\sup _{n \in \boldsymbol{N}}\left|a_{n} n !\right| . \quad$ If $f \in P$ and $t \in \boldsymbol{R}, f_{t}$ denotes the function $x \rightarrow$ $f(x+t)$.

Lemma 4. 1: $f_{t} \in P$ for any $f \in P$ and $t \in \boldsymbol{R}$. Moreover, the mapping $\eta:\left\{\begin{array}{l}\boldsymbol{R} \times P \rightarrow P \\ (t, f) \rightarrow f_{t}\end{array}\right.$ is smooth.

Proof: Let $f$ be given by $f(x)=\sum a_{n} x^{n}$. Then

$$
f_{t}(x)=\sum_{n \in N} \sum_{k=0}^{\infty} a_{n} \frac{n !}{(n-k) ! k !} x^{k} t^{n-k}=\left(\sum_{n=k}^{\infty} a_{n} \frac{n ! t^{n-k}}{(n-k) ! k !}\right) x^{k},
$$

so that

$$
\left\|f_{t}\right\| \leqslant \sup _{k \in \boldsymbol{N}}\left(\sum_{n=k}^{\infty}\left|a_{n}\right| \frac{n !}{(n-k) !}|t|^{n-k}\right) \leqslant \sup _{l \in \boldsymbol{N}}\left|a_{l} l !\right| \cdot \sum_{n=0}^{\infty} \frac{|t|^{n}}{n !}=\|f\| e^{|t|},
$$

which proves the first assertion. Let $f^{(m)}$ denote the $m$-th derivative of $f$. It is easy to check that $f \in P$ implies $f^{(m)} \in P$ and $\left\|f^{(m)}\right\| \leqslant\|f\|$ for all $m \in N$. Let $\varphi_{k}(f, s)$ be the function $z \rightarrow$ $f(z+s)-\sum_{m=0}^{k} \frac{1}{m !} f^{(m)}(z) s^{m} ;$ a direct computation shows that the right-hand term is equal to

$$
s^{k+1} \sum_{j=0}^{\infty} \frac{1}{j !}\left(\sum_{i=0}^{\infty} \frac{(i+j+k+1) ! a_{i+j+k+1}}{(i+k+1) !} s^{i}\right) z^{j}
$$

so that $\left\|\varphi_{k}(f, s)\right\| \leqslant|s|^{k+1} e^{|s|}\|f\|$. Let now $\psi_{k}(f, t+s)$ be the function $x \rightarrow f(x+t+s)-\sum_{m=0}^{k-1} \frac{1}{m !} f^{(m)}(x+t) s^{m} ;$ similarly: $\left\|\psi_{k}(f, t+s)\right\|$ $\leqslant|s|^{k} e^{|t|+|s|}\|f\|$. We claim now that $\left(D^{k} \eta\right)_{(t, f)}\left(s_{1}, f_{1}\right) \cdots\left(s_{k}, f_{k}\right)$ is the function which takes at $x$ the value

$$
f^{(k)}(x+t) s_{1} \cdots s_{k}+\sum_{j=1}^{k} f_{j}^{(k-1)}(x+t) s_{1} \cdots \hat{s}_{j} \cdots s_{k} .
$$

It is sufficient to check (see [15] p. 7) that if $\delta=\sqrt{|s|^{2}+\|g\|^{2}}$ one has $\lim _{\delta \rightarrow 0} \frac{1}{\delta^{k}}\|F\|=0$, where $F$ is the function

$$
x \rightarrow(f+g)(x+t+s)-\left\{\sum_{m=0}^{k} \frac{1}{m !} f^{(m)}(x+t) s^{m}+\sum_{m=0}^{k-1} \frac{1}{m !} g^{(m)}(x+t) s^{m}\right\} ;
$$

but this follows directly from the inequalities computed above. 
Lemma 4. 2: Let $\xi$ be the $\operatorname{map}\left\{\begin{array}{l}P \times \boldsymbol{R} \rightarrow \boldsymbol{R} \\ (f, x) \rightarrow f(x)\end{array}\right.$. Then $\xi$ is smooth.

Proof: Let $f, g \in P ; x, y \in \boldsymbol{R}$. Consider the number

$$
\bigwedge=(f+g)(x+y)-\sum_{m=0}^{k} \frac{1}{m !} f^{(m)}(x) y^{m}-\sum_{m=1}^{k} \frac{1}{(m-1) !} g^{(m-1)}(x) y^{m-1} \text {. }
$$

Let $\delta=\sqrt{\|g\|^{2}+|y|^{2}}$; it is easy to show that $\lim _{\delta \rightarrow 0} \frac{|\wedge|}{\delta^{k}}=0$. In other words $\left(D^{k} \xi\right)_{(f, x)}\left(f_{1} y_{1}\right) \cdots\left(f_{k} y_{k}\right)$ exists and is given by $f^{(k)}(x) y_{1} \cdots y_{k}$ $+\sum_{m=1}^{k} f_{j}^{\left({ }^{k-1)}\right.}(x) y_{1} \cdots \hat{y}_{j} \cdots y_{k}$ for all $k \in \mathbf{N}$.

Define now a multiplication on the space $G=\boldsymbol{R} \oplus P$ by $(t, f)\left(t_{1}, f_{1}\right)$ $=\left(t+t_{1}, f_{t}+f_{1}\right)$; this makes $G$ a Banach-Lie group with unit $(0,0)$; the inverse of an element $(t, f)$ is $\left(-t,-f_{-t}\right)$; Lemma 4.1 shows that the group operations are smooth. Define an action of $G$ on $\boldsymbol{R}^{2}$ by $(t, f)(x, y)=(t+x, f(x)+y)$; it is readily checked that it is indeed an action, which is smooth in virtue of Lemma 4.2. Let $(t, f) \in G$ with $(t, f)(x, y)=(x, y)$ for all $(x, y) \in \boldsymbol{R}^{2}$; then $(t, f)$ $=(0,0)$, i.e. the action is effective. Let $(x, y) \in \boldsymbol{R}^{2}$ and let $f \in P$ such that $f(0)=y$; then $(x, f)(0,0)=(x, y)$, i.e. the action is transitive. Finally, it is a very easy excercise to show that the action is ample. In confirmation of Theorem $\mathrm{I}$, this action is however not primitive. Indeed, let $G_{0}$ be the isotropy group of the point $(0,0)$ in $R^{2}$, and consider $P$ as a subgroup of $G$; then:

$$
G_{0} \subsetneq P \varsubsetneqq G \text {. }
$$

We sum up this discussion as follows.

Example 4. 3: $G$ is an infinite dimensional Banach-Lie group which acts smoothly, effectively, amply and not primitively on the finite dimensional $\boldsymbol{R}^{2}$.

\section{Classical Banach-Lie groups of operators}

Let $H$ be a separable infinite dimensional complex Hilbert space. 
Let $L(H)$ be the associative Banach algebra of continuous linear operators in $H$; let $\mathcal{C}(H)$ be the ideal of compact operators in $H$ and let $L^{\circ} \mathrm{C}(H)$ be the (non-closed) ideal of finite rank operators in $H$. We first recall three classical results.

Lemma 5.1 (Calkin). Let $\mathcal{L}$ be a non-trivial two-sided ideal in $L(H)$. Then $L^{\circ} \mathcal{C}(H) \subset \mathcal{L} \subset \mathcal{C}(H)$. In particular, any nontrivial ideal in $L(H)$ is infinite codimensional, the only non-trivial closed ideal in $L(H)$ is $\mathcal{C}(H)$, and the associative algebra $L(H)$ $/ \mathcal{C}(H)$ is simple.

Proof: See [3] Th. 1.4 and 1.7, or [22] Chap. I $\S 6$ Theorem 11.

The aim of this section is to point out analogous results for various Banach-Lie algebras. Each of them will fulfill the conditions of validity of Theorem II.

Lemma 5.2 (Schur). Let $X \in L(H)$ and let $S$ be a subset of $L(H)$ which has no non-trivial closed invariant subspace. Suppose $X$ is normal and commutes with all the elements of $S$. Then $X$ is a multiple of the identity.

Proof: Via spectral theorem; see e.g. [13] Appendice II §2.

Lemma 5.3 (Calkin). The center of the algebra $L(H) / C(H)$ consists exactly of the multiples of the identiy.

Proof: See [3] Th. 2.9.

\section{A. General linear Lie algebras}

We denote by $\mathfrak{g l}(H)$ the Banach-Lie algebra of the associative Banach algebra $L(H)$, by $\mathfrak{g l}(H ; \mathcal{C})$ that of $\mathcal{C}(H)$, and by $\mathfrak{g l}\left(H ; L^{\circ} \mathcal{C}\right)$ the Lie algebra of the associative algebra $L^{0} \mathcal{C}(H)$. Let $\mathfrak{g l}(H ; C i d+C)$ be the Banach-Lie algebra $\{X \in \mathfrak{g l}(H) \mid$ there exists $\lambda \in C$ and $Y$ 
$\in \mathcal{C}(H)$ with $X=\lambda I+Y\}$ and let $\mathfrak{g l}\left(H ; L^{\circ} \mathcal{C}\right)$ be the Lie algebra of zero-trace operators in $\mathrm{gl}\left(H ; L^{0} \mathrm{C}\right)$.

Lemma 5.4. A. The Lie algebra $\mathfrak{g l}\left(H ; L^{0} \mathcal{C}\right)$ is simple.

Proof: It is clearly sufficient to prove that $\operatorname{sl}\left(H ; L^{\circ} \mathrm{C}\right)$ is locally simple, i.e. that any finite subset of it is contained in a simple finite dimensional Lie algebra. Let $\left\{X_{j}\right\}_{1 \leqslant j \leqslant n}$ be a finite subset of $\mathfrak{g l}\left(H ; L^{0} \mathcal{C}\right)$. For each $j, \operatorname{im}\left(X_{j}\right)+\operatorname{im}\left(X_{j}^{*}\right)$ is finite dimensional, and $\operatorname{ker}\left(X_{j}\right) \cap \operatorname{ker}\left(X_{j}^{*}\right)$ is finite codimensional; hence there exists a finite dimensional subspace $F$ in $H$ such that

$$
\bigcup_{j=1}^{n}\left(\operatorname{im}\left(X_{j}\right) \cup \operatorname{im}\left(X_{j}^{*}\right)\right) \subset F \text { and } F^{\perp} \subset \bigcap_{j=1}^{\infty}\left(\operatorname{ker}\left(X_{j}\right) \cap \operatorname{ker}\left(X_{j}^{*}\right)\right) .
$$

Hence $X_{j}, X_{j}^{*} \in \mathfrak{g l}(F)$ for $j=1, \cdots, n$, where the simple Lie algebra $\mathfrak{g l}(F)$ is identified with a subalgebra of $\mathfrak{g l}\left(H ; L^{0} \mathcal{C}\right)$ in the obvious way.

N.B.: The only reason for which we have introduced the $X_{j}^{*}$ 's in the proof is that the same proof will so work below for the cases $\mathrm{B}$ and $\mathrm{C}$.

Lemma 5. 5. A. Let a be a Lie ideal in $\mathrm{gl}(H)$ which is neither $\{0\}$ nor $\{\lambda I \mid \lambda \in C\}$. Then $\mathfrak{3 l}\left(H ; L^{\circ} \mathcal{C}\right) \subset a$.

Proof: Choose $X \in \mathfrak{a}, X$ is not a multiple of the identity. One can suppose without loss of generality that $X$ is normal (if not, take $\left.\left[X, X^{*}\right]\right)$. By Lemma 5.2, there exists $Y \in L^{0} \mathcal{C}(H)$ such that $[X, Y] \neq 0$; hence $\mathfrak{a} \cap \mathfrak{g l}\left(H ; L^{0} \mathcal{C}\right) \neq\{0\}$. The conclusion follows from Lemma 5. 4. A.

Lemma 5.6. A (Halmos). Any operator in $L(H)$ can be written as the sum of two commutators.

Proof: See [5] Chap. XV $\S 11$ exercise 23, or references given in [2] Chap. I. 
Lemma 5. 7. A (Herstein), Let $\mathcal{R}$ be a simple associative real algebra with center $\mathcal{L}$. Let a be a Lie-ideal in $\mathcal{R}$. Then one has either $a \subset \mathcal{L}$ or $[R, R] \subset a$.

Proof: See [8] Theorem 4. Suppose $\mathcal{R}$ has the following property; for any $Y \in \mathscr{R}-\{0\}$, there exists $T \in \mathscr{R}$ such that $Y T$ is not nilpotent; then there is a much shorter proof than the one given in [8], which is indicated in [1] Chap. I $\S 1$ exercise 7. That $L(H) / C(H)$ has this property is clear from the well-known characterisation: for $X \in L(H), X$ is compact if and only if the image $X(H)$ contains no infinite dimensional clused subspace of $H$.

Theorem IV A. Let a be a non-trivial Lie ideal of $\mathfrak{g l}(H)$. Then either $\mathfrak{a}=\{\lambda I \mid \lambda \in C\}$ or $\mathfrak{s l}\left(H ; L^{\circ} \mathcal{C}\right) \subset \mathfrak{a} \subset \mathfrak{g l}(H ; C i d+C)$. In particular, any non-trivial ideal of $\mathrm{gl}(H)$ is infinite codimensional; the only non-trivial closed ideals of $\mathfrak{g l}(H)$ are $\{\lambda I \mid \lambda \in C\}, \mathfrak{l l}(H ; \mathcal{C})$ and $\operatorname{gl}(H ; C i d+C)$; and the Banach-Lie algebra $\operatorname{gl}(H) / \mathfrak{g l}(H$; Cid $+\mathcal{C})$ is algebraically simple.

The proof is clear from the preceeding lemmas. We insist on the distinction between the Banach-Lie algebra $\operatorname{gl}(H ; \mathcal{C})$ which is (topologically) simple, i.e. which has no non-trivial closed ideals, and the Banach-Lie algebra $\mathfrak{g l}(H) / \mathfrak{g l}(H ; \boldsymbol{C} i d+C)$ which is algebraically simple. $g l(H)$ being the Lie algebra of the Banach-Lie group $G L(H)$, the Theorems I and II apply.

\section{B. Orthogonal Lie algebras}

Recall that a conjugation in $H$ is a semi-linear operator $J_{R}$ in $H$ such that $J_{R}^{2}=i d$ and $\left\langle J_{R} x \mid J_{R} y\right\rangle=\overline{\langle x \mid y\rangle}$ for all $x, y \in H$. It is then easy to show that there exists an orthonormal basis $\left(e_{n}\right)_{n \in N}$ of $H$ such that $J_{R}\left(\sum_{n \in N} x_{n} e_{n}\right)=\sum_{n \in N} \bar{x}_{n} e_{n}$. In particular, any two conjugations in $H$ are conjugate to each other by unitary operators. From now on, we choose a fixed conjugation $J_{R}$ in $H$. Let $\varphi_{R}$ be the map 
$\left\{\begin{array}{c}L(H) \rightarrow L(H) \\ X \rightarrow J_{\boldsymbol{R}} X^{*} J_{\boldsymbol{R}}\end{array} ; \varphi_{\boldsymbol{R}}\right.$ is clearly an isometric involutive antiautomorphism of the associative Banach algebra $L(H)$, which keeps the identity, $\mathcal{C}(H), L^{\circ} \mathcal{C}(H)$ and $\left\{X \in L^{\circ} \mathcal{C}(H) \mid \operatorname{trace}(X)=0\right\}$ invariant. Abusively, we denote still by $\varphi_{\boldsymbol{R}}$ the involutions induced on invariant subsets of $L(H)$ and on $L(H) / C(H)$. We consider now the orthogonal complex Banach-Lie algebras $\mathfrak{O}\left(H, J_{R}\right)=\left\{X \in \mathfrak{g l}(H) \mid \varphi_{R}(X)\right.$ $=-X\} \quad \mathfrak{O}\left(H, J_{R} ; \mathcal{C}\right)=\left\{X \in \mathfrak{g l}(H ; \mathcal{C}) \mid \varphi_{R}(X)=-X\right\}$ and the Lie algebra $\mathfrak{S O}\left(H, J_{R} ; L^{0} \mathcal{C}\right)=\left\{X \in \mathfrak{g l}\left(H ; L^{0} \mathcal{C}\right) \varphi_{R}(X)=-X\right\}$.

Lemma 5.4. B. The Lie algebra $\mathfrak{s}_{0}\left(H, J_{R} ; L^{0} \mathcal{C}\right)$ is simple.

Proof: With the same notations as in the proof of Lemma 5.4. B, note that $\operatorname{im}\left(X_{j}\right)+\operatorname{im}\left(X_{j}^{*}\right)$ and $\operatorname{ker}\left(X_{j}\right) \cap \operatorname{ker}\left(X_{j}^{*}\right)$ are invariant by $J_{R}$; chose $F$ invariant by $J_{R}$ and proceed as before.

Lemma 5.5. B. Let a be a non-zero ideal of $\subseteq\left(H, J_{R}\right)$. Then $\mathfrak{S o}\left(H, J_{R} ; L^{\circ} \mathcal{C}\right) \subset a$.

Proof: As above; note that $\mathcal{O}\left(H, J_{R}\right)$ does not contain the identity.

Lemma 5. 6. B. Any operator in $\mathfrak{O}\left(H, J_{R}\right)$ can be written as a finite sum of commutators of operators each in $\mathfrak{O}\left(H, J_{R}\right)$.

Proof: In three steps.

Step one: Let $B$ be a set of generators in an associative algebra $\mathcal{R}$, then $[B, \mathcal{R}]=[R, \mathscr{R}]$. We first prove by induction that $\left[B^{n}, \mathcal{R}\right] \subset[B, \mathcal{R}]$ for any $n \in N^{*}$; it is trivial for $n=1$; suppose it is true for $i=1,2, \cdots, n-1$; choose $X_{1}, \cdots, X_{n} \in B$ and $Y \in \mathscr{R}$; then:

$$
\begin{aligned}
& {\left[X_{1} X_{2} \cdots X_{n}, Y\right]=X_{1}\left(X_{2} \cdots X_{n} Y\right)-\left(X_{2} \cdots X_{n} Y\right) X_{1}} \\
& \quad+X_{2} \cdots X_{n}\left(Y X_{1}\right)-\left(Y X_{1}\right) X_{2} \cdots X_{n} \in[B, \mathcal{R}]+\left[B^{n-1}, \mathcal{R}\right] \subset[B, \mathcal{R}] .
\end{aligned}
$$

Now $[R, R] \subset \sum_{n \in N^{*}}\left[B^{n}, \mathscr{R}\right] \subset[B, \mathscr{R}]$ and step one is established. 
Step two: $\mathfrak{S}\left(H, J_{\boldsymbol{R}}\right)$ is a set of generators in $L(H)$. See [9] Theorem 15.

Step three: $\quad\left[\mathfrak{O}\left(H, J_{\boldsymbol{R}}\right), \mathfrak{O}\left(H, J_{\boldsymbol{R}}\right)\right]=\mathfrak{O}\left(H, J_{\boldsymbol{R}}\right)$. Let $\mathfrak{m}=\{X$ $\left.\in \mathfrak{g l}(H) \mid \varphi_{\boldsymbol{R}}(X)=X\right\}$; then $\mathfrak{g l}(H)=\mathfrak{O}\left(H, J_{\boldsymbol{R}}\right) \oplus \mathrm{m}$. By Lemma 5. 6. A and the two first steps, one has:

$$
\begin{aligned}
\mathfrak{g l}(H) & =[\mathfrak{g l}(H), \mathfrak{g l}(H)]=\left[\mathfrak{S}\left(H, J_{\boldsymbol{R}}\right), \mathfrak{O}\left(H, J_{\boldsymbol{R}}\right) \oplus \mathfrak{m}\right] \\
& \subset\left[\mathfrak{O}\left(H, J_{\boldsymbol{R}}\right), \mathfrak{O}\left(H, J_{\boldsymbol{R}}\right)\right]+\left[\mathfrak{O}\left(H, J_{\boldsymbol{R}}\right), \mathfrak{m}\right] .
\end{aligned}
$$

As $\left[\mathfrak{O}\left(H J_{\boldsymbol{R}}\right), \mathfrak{O}\left(H, J_{\boldsymbol{R}}\right)\right] \subset \mathfrak{D}\left(H, J_{\boldsymbol{R}}\right)$ and $\left[\mathfrak{O}\left(H, J_{\boldsymbol{R}}\right), \mathfrak{m}\right] \subset \mathfrak{m}$ the conclusion follows.

Lemma 5. 7. B (Herstein). Let $\mathscr{R}$ be a simple associative real algebra with center $\mathcal{L}$. Suppose that the dimension of $\mathscr{R}$ over $\mathcal{L}$ is larger than 16. Let $\varphi$ be an involutive antiautomorphism of $R$ and consider the Lie algebra $\mathfrak{O}=\{X \in \mathscr{R} \mid \varphi(X)=-X\}$. Let a be a Lie ideal in $\mathfrak{O}$. Then one has either $a \subset \mathcal{L}$ or $[\mathfrak{O}, \mathfrak{O}]$ a.

Proof: See [9] Theorem 26. In fact, Herstein proves a much more general result, where $\mathcal{R}$ can be any ring; we haven't tried to find a shorter proof for the case $R=L(H)$.

Theorm IV B. Let a be a non-trivial Lie ideal of $\mathfrak{O}\left(H, J_{\boldsymbol{R}}\right)$. Then $\mathfrak{s} \mathfrak{D}\left(H, J_{R} ; L^{0} \mathcal{C}\right) \subset a \subset\left(H, J_{R} ; \mathcal{C}\right)$. In particular, any non-trivial ideal of $\bigcirc\left(H, J_{R}\right)$ is infinite codimensional, the only non-trivial closed ideal of $\mathfrak{O}\left(H, J_{\boldsymbol{R}}\right)$ is $\mathfrak{O}\left(H, J_{\boldsymbol{R}} ; C\right)$, and the Banach-Lie algebra $\mathfrak{O}\left(H, J_{R}\right) / \mathfrak{O}\left(H, J_{R} ; \mathcal{C}\right)$ is algebraically simple.

$\bigcirc\left(H, J_{R}\right)$ is the Lie algebra of the Banach-Lie group $O\left(H, J_{R}\right)$ so that Theorems I and II apply. $O\left(H, J_{R}\right)$ is the closed sub BanachLie group and submanifold of $G L(H)$ given as follows: let $\{\mid\}$ be the bilinear from defined on $H$ by $\{y \mid z\}=\left\langle y \mid J_{\boldsymbol{R}} z\right\rangle$; then $O\left(H, J_{\boldsymbol{R}}\right)$ $=\{X \in G L(H) \mid\{X y \mid X z\}=\{y \mid z\}$ for all $y, z \in H\}$. 


\section{C. Symplectic Lie algebras}

Recall that an anticonjugation in $H$ is a semi-linear operator $J_{Q}$ in $H$ such that $J_{Q}^{2}=-i d$ and $\left\langle J_{Q} x \mid J_{Q} y\right\rangle=\overline{\langle x \mid y\rangle}$ for all $x, y \in H$. It is then easy to show that there exists an orthonormal basis $\left(e_{n}\right)_{n \in \boldsymbol{Z}^{*}}$ of $H$ such that $J_{\boldsymbol{Q}}\left(\sum_{n \in N^{*}} x_{-n} e_{-n}+\sum_{n \in \boldsymbol{N}^{*}} x_{n} e_{n}\right)=\sum_{n \in \boldsymbol{N}^{*}} \bar{x}_{-n} e_{n}-\sum_{n \in \boldsymbol{N}^{*}} \bar{x}_{n} e_{-n}$. In particular, any two anticonjugations in $H$ are conjugate to each other by unitary operators. From now on, we choose a fixed anticonjugation $J_{\boldsymbol{Q}}$ in $H$.

Let $\varphi_{\boldsymbol{Q}}$ be the antiautomorphism $X \rightarrow-J_{\boldsymbol{Q}} X^{*} J_{\boldsymbol{Q}}$ of $L(H)$. We consider the symplectic complex Banach-Lie algebras $\mathfrak{a p}\left(H, J_{\boldsymbol{Q}}\right)=\{X$ $\left.\left.\in \mathfrak{g l}(H)\right|_{\boldsymbol{Q}}(X)=-X\right\} \quad \mathfrak{g} \mathfrak{p}\left(H, J_{\boldsymbol{Q}} ; \mathcal{C}\right)=\left\{X \in \mathfrak{g l}(H ; \mathcal{C}) \mid \varphi_{\boldsymbol{Q}}(X)=-X\right\}$ and the Lie algebra $\mathfrak{a p}\left(H, J_{\boldsymbol{Q}} ; L^{0} \mathcal{C}\right)$. Lemmas 5.4.C to 5.7.C are as in section $5 . \mathrm{B}$, and one has the following theorem.

Theorem IV C. Let a be a non-trivial Lie ideal of $\mathfrak{s p}\left(H, J_{\boldsymbol{Q}}\right)$. Then $\mathfrak{s p}\left(H, J_{\boldsymbol{Q}} ; L^{0} \mathcal{C}\right) \subset \mathfrak{a} \subset \mathfrak{s p}\left(H, J_{\boldsymbol{Q}} ; \mathcal{C}\right)$. In particular, any nontrivial ideal of $\mathfrak{s}\left(H, J_{\boldsymbol{Q}}\right)$ is infinite codimensional, the only nontrivial closed ideal of $\mathfrak{a p}\left(H, J_{\boldsymbol{Q}}\right)$ is $\mathfrak{a p}\left(H, J_{\boldsymbol{R}} ; \mathcal{C}\right)$, and the BanachLie algebra $\mathfrak{s p}\left(H, J_{\boldsymbol{Q}}\right) / \mathfrak{a p}\left(H, J_{\boldsymbol{Q}} ; \mathcal{C}\right)$ is algebraically simple.

$\mathfrak{g p}\left(H, J_{\boldsymbol{Q}}\right)$ is the Lie algebra of the Banach-Lie group $\operatorname{Sp}\left(H, J_{\boldsymbol{Q}}\right)$, so that Theorems I and II apply. $S p\left(H, J_{\boldsymbol{Q}}\right)$ is the closed sub Banach-Lie group and submanifold of $G L(H)$ given as follows: let $\{\mid\}$ be the bilinear form defined on $H$ by $\{y \mid z\}=\left\langle y \mid J_{\boldsymbol{Q}} z\right\rangle$; then $S p\left(H, J_{\boldsymbol{Q}}\right)=\{X \in G L(H) \mid\{X y \mid X z\}=\{y \mid z\}$ for all $y, z \in H\}$.

N.B.: $\mathfrak{g l}(H), \mathfrak{O}\left(H, J_{\boldsymbol{R}}\right)$ and $\mathfrak{a p}\left(H, J_{\boldsymbol{Q}}\right)$ can naturally be considered as real algebras, and so can the corresponding Lie groups, by restriction of the scalars.

\section{R. Real forms}

Let $\mathrm{g}$ be one of the complex Lie algebra of operator described above. A conjugation in $\mathfrak{g}$ is a map $\tau: \mathfrak{g} \rightarrow \mathfrak{g}$ such that the map 
$X \rightarrow \tau\left(-X^{*}\right)$ is an isomorphism. A real form of $g$ is an $\boldsymbol{R}$-sub-Lie algebra $\mathfrak{g}_{0}$ of $\mathfrak{g}$ such that $\mathfrak{g}=\mathfrak{g}_{0} \oplus i g_{0}$. It is well known that one can associate a conjugation to any real form and vice-versa.

To any non-exceptional class of finite dimensional simple real Lie algebras with simple complexifications, one can associate a conjugation of one of $\mathfrak{g l}(H), \mathfrak{O}\left(H, J_{R}\right)$ and $\mathfrak{s p}\left(H, J_{\boldsymbol{Q}}\right)$. This procedure has been described in detail elsewhere (see [7] and references there), and we will not repeat it here; we only mention the following result:

Theorem IV R. Let a be an ideal of $g_{0}$, where $g_{0}$ is a real form as described above. Then a is infinite codimensional. Theorems $I$ and II apply to the Lie group corresponding to $\mathrm{g}_{0}$.

We suggest the following definitions. By a classical complex Banach-Lie group of bounded operators in $H$, we mean one of the three groups $G L(H), O\left(H, J_{\boldsymbol{R}}\right)$ and $S p\left(H, J_{\boldsymbol{Q}}\right)$. By a classical real Banach-Lie group of bounded operators in $H$, we mean either one of these same three groups after restriction of the scalars, or one of the groups attached to a real form $g_{0}$ as above; the list of these can be given as follows, using the notations of [7]:

Type A I $G L\left(E_{R}\right)$

A II $\quad U^{*}(E)$

A III $U(E, r, \infty)$ with $r \in N \cup\{\infty\}$

$U(E)$ corresponds to $r=0$

Type BD I $O(E, r, \infty)$ with $r \in \boldsymbol{N} \cup\{\infty\}$

$O\left(E_{R}\right)$ corresponds to $r=0$

BD III $O^{*}(E)$

Type C I $S p(E, \boldsymbol{R})$

C II $\quad S p(E, r, \infty)$ with $r \in \boldsymbol{N} \cup\{\infty\}$

$S p\left(E_{\boldsymbol{Q}}\right)$ correponds to $r=0$

This makes precise the content of Theorem IV, as written in the introduction. 
By a classical Banach-Lie group of compact operators in $H$ we mean one obtained as follows. Let $G(H)$ be a classical BanachLie group of bounded operators in $H$, and let $\mathfrak{g}(H)$ be its Lie algebra. Let $g\left(H, L^{0} \mathrm{C}\right)$ be the Lie algebra $g(H) \cap \mathfrak{g l}\left(H ; L^{0} \mathrm{C}\right) ; \mathrm{g}\left(H ; L^{0} \mathrm{C}\right)$ is simple. Let $\alpha$ be a uniform crossnorm in the sense of [22], and let $\mathrm{g}(H, \alpha)$ be the completion of $\mathrm{g}\left(H ; L^{0} \mathcal{C}\right)$ with respect to $\alpha$. Then $\mathrm{g}(H, \alpha)$ is the Lie algebra of a sub Banach-Lie group of $G(H)$ which is denoted by $G(H, \alpha) . G(H, \alpha)$ is by definition a classical BanachLie group of compact operators in $H$, and satisfies the conditions of Theorems I and II. Note that if $\alpha$ is the uniform crossnorm, $G(H, \alpha)$ $=G(H, \mathcal{C}) ;$ if $\alpha$ is the Hilbert-Schmidt cross-norm, $G(H, \alpha)=G(H$, $\left.L^{2} \mathcal{C}\right)$ is a $L^{*}$-group [7].

\section{References}

[1] N. Bourbaki: Groupes et algèbres de Lie, Chap. I. Hermann 1960.

[2] D. Burghelea and N. H. Kuiper: Hilbert manifolds. Ann Math 90 (1969) 379417.

[3] J. W. Calkin: Two-sided ideals and congruences in the ring of bounded operators in Hilbert space. Ann of Math 42 (1941) 838-873.

[4] J. Dieudonné: Les fondements de l'analyse moderne. Gauthier-Villars 1963.

[5] : Eléments d'analyse, tome 2. Gauthier-Villars 1968.

[6] D. G. Ebin and J. Marsden: Groups of diffeomorphisms and the motion of an incompressible fluid. Ann of Math. 92 (1970) 102-163.

[7] P. de la Harpe: Classification des $L^{*}$-algèbres semi-simples réelles séparables. CR Acad. Sci. Paris. Ser. A 272 (1971) 1559-1561.

[8] L. N. Herstein: On the Lie and Jordan Rings of a simple associative ring. Am. J. Math. 77 (1955) 279-285.

[9] - Lie and Jordan systems in simple rings with involution. Am. J. Math. 78 (1956) 629-649.

[10] : Topics in Ring Theory. University of Chicago, Math. Lect. Notes, 1965.

[11] S. Kobayashi and T. Nagano: A report on filtered Lie algebras. Proc. of the US-Japan Sem. in Diff. Geom., Kyoto 1965.

[12] — On filtered Lie algebras and geometric strures III, IV. J. of Math. and Mech. 14 (1965) 679-706 and 15 (1966) 163-175.

[13] S. Lang: Introduction aux variétés diffrentiables. Dunod 1967.

[14] J. A. Leslie: On a differential structure for the group of diffeomorphisms. Topology 6 (1967) 263-271.

[15] E. Nelson: Topics in dynamics I: Flows. Math. Notes, Princeton Press, 1969.

[16] H. Omori: On the group of diffeomorphisms on a compact manifold. Proc. Symp. Pure Math. AMS 15 (1970) 167-183.

[17] : Local structures of groups of diffeomorphisms. Jour. Math. Soc. 
Japan 24 (1972) pp. 60-80.

[18] : On smooth extension theorems. Jour. Math. Soc. Japan 24 (1972) pp. $405-432$.

[19] - and P. de la Harpe: Opération de groupes de Lie banachiques sur des varitétés différentielles de dimension finie. CR Acad. Sci. Paris. Ser. A (19 juillet 1971).

[20] R, Ouzilou: Groupes classiques de Banach. CR Acad. Sci. Paris, Ser. A 272 (1971) 1459-1461.

[21] C. R. Putnam: Commutation properties of Hilbert space operators and related topics. Springer 1967.

[22] R. Schatten: Norm ideals of completely continuous operators. Springer 1960.

[23] I. M. Singer and S. Sternberg: The infinite groups of Lie and Cartan, part I (The transitive groups). Journ. d'an. Math. 15 (1965) 1-114.

[24] T. Morimoto and N. Tanaka: The classification of the real primitive infinite Lie algebras. J. Math. Kyoto Univ. 10 (1970) 207-243.

Mathematics Institute

UNIVERSITY OF WARWICK

Conventry (G. B.) 